\title{
On the Current State of Powder Characterization
}

\author{
Joseph W. Newkirk and Caitlin S. Kriewall
}

Department of Materials Science, Missouri University of Science and Technology, Rolla, Mo, USA

Modern powder metallurgy (PM) has been a prominent field since the 1920s when press-and-sintering offered a fast, efficient way to produce mass quantities of parts [1]. Characterization of the starting powder has always been of significant importance, because the powder properties influence its behavior during processing and, therefore, the final part. There has been unprecedented growth in PM in the past two decades due to new technologies such as metal injection molding (MIM) and additive manufacturing (AM). These new technologies have attracted the interest of different industries, such as automotive and aerospace, who are considering the use of PM for more critical parts. Thus, more strenuous characterization of the starting powder material as well as the built parts is required [2].

Powder morphology remains an essential parameter to characterize because it directly affects how the particles interact together. Scanning electron microscopy (SEM) or optical microscopy continue to dominate the study of powder morphology [3], [4]. This is due to the ability to capture a static image and manipulate it with image analysis software in order to calculate dimensional descriptions of the powder shape. Qualitative descriptions of powder particles are often used and are standardized [5]. However, there is a great need for quantitative descriptions. Many descriptions and ratios have been implemented in the characterization of powder morphology, but no standard has been created. These quantitative descriptions of powder morphology are discussed in more detail in references [6]-[8]

Characterization of the particle size distribution is essential to understanding the behavior of powders and different processes require very different distributions. Sieve analysis is an industrial standard for size distributions, however it is reserved for applications that use powders with particles larger than 45 $\mu \mathrm{m}$ [9]. There are other techniques that are more widely used for the analysis of powders in the smaller size range. Counting and measuring particles from SEM micrographs is a reliable method for researchers, although the post-processing is time consuming. SEMs equipped with automated feature analysis have offered the advantage of reducing the time for analyzing these micrographs. There is, however, a lack of agreement on the minimum number of particles needed in order for these results to be acceptable [3]. Particle size analyzers is often used as well. Laser diffraction is a technique that can analyze the full size range $(0.4 \mu \mathrm{m}$ to $2 \mathrm{~mm})$ of powders for most PM applications [6].

The determination of flowability and density are very important. Flowability is measured by funnel techniques and the angle of repose [2], [10]. However, there is a need for more thorough characterization of flowability. Powder rheometers are being used in an attempt to fully characterize the flowability [11], [12]. Powder rheometers implement several testing methods in an attempt to simulate the interactions that powders will have before and during processing. These tests have been shown to detect differences in powders that the traditional flowability methods failed to pinpoint. Details about these methods are described in reference [12].

The apparent, tap, and true densities of powders continue to be important characteristics to determine [13]-[17]. True, or skeletal, density measurements are accomplished using gas pycnometry which is able to determine the volume of the sample (taking into account porosity) and the density is subsequently 
calculated [18]. Further characterization of the porosity can be accomplished using mercury porosimetry or BET (gas adsorption) [2].

As the chemistry of the powder can significantly affect the properties of the final part, it is vital to comprehensive characterization. The bulk chemistry methods most widely used are x-ray fluorescence, inductively coupled plasma optical emission spectroscopy and atomic absorption spectroscopy, although techniques such as inert gas fusion and high temperature oxidation are also available for the light elements in metals. These techniques are discussed in more detail elsewhere [2],[6].

Surface chemistry is also significant as the powders interact through their surfaces. X-ray photoelectron spectroscopy and auger electron spectroscopy are recommended techniques to accomplish this task (depth of analysis is less than $3 \mathrm{~nm}$ ). However, these high vacuum techniques could alter the surface chemistry which interparticle friction is controlled by. Surface sensitive techniques which do not dry or desorb gases from the particle surfaces are being developed. Infrared or lower frequency radiation interacts with surface chemical species without penetrating the metal and may offer advantages over other techniques. Analysis of individual particles or powder masses may be possible allowing both qualification and process feedback.

Despite current characterization methods, there is still a noted disconnect between these methods and powder behavior. For example in AM, powders with identical measured characteristics often do not perform the same. This is significant in that quality standards cannot be written based on powder measurables. New techniques continue to be developed which can better define the differences between powder lots. The need for comprehensive characterization as well as an outline of certain pass-or-fail criteria for powders that are analyzed is necessary. With the continued expansion of PM technologies, the characterization of the starting powder material will continue to see growth in the upcoming years.

\section{References:}

[1] G. S. Upadhyaya, Powder Metallurgy Technology. Cambridge International Science Pub., 2002.

[2] P. K. Samal and J. W. Newkirk, Eds., ASM Handbook Volume 7: Powder Metallurgy. 2015.

[3] D. O. C. Souza and F. C. Menegalli, Powder Technol., vol. 214, no. 1, pp. 57-63, 2011.

[4] S. Almeida-Prieto, et al.., Eur. J. Pharm. Biopharm., vol. 67, no. 3, pp. 766-776, 2007.

[5] ASTM B243-13, 2014.

[6] A. Cooke and J. Slotwinski, NISTIR, 2012.

[7] M. Allen, G. J. Brown, and N. J. Miles, Powder Technol., vol. 84, no. 1, pp. 1-14, 1995.

[8] E. Mikli Valdek, et al., Methods, 2001, vol. 7, pp. 22-34.

[9] ASTM F3049 - 14, 2014.

[10] ASTM B213-13, 2014.

[11] A. Strondl, O. Lyckfeldt, H. Brodin, and U. Ackelid, JOM, vol. 67, no. 3, pp. 549-554, 2015.

[12] M. Delancy, AMPM Conference Proceedings, 2014.

[13] ASTM B703-10, 2014.

[14] ASTM B417-13, 2013.

[15] ASTM B329-14, 2014.

[16] ASTM B212-13, 2014.

[17] ASTM B527-15, 2015.

[18] ASTM B923-10, 2015. 